\title{
THREE EXAMPLES OF THREE-DIMENSIONAL CONTINUED FRACTIONS IN THE SENSE OF KLEIN.
}

\author{
OLEG KARPENKOV
}

The problem of investigation of the simplest $n$-dimensional continued fraction for $n \geq 2$ was posed by $\mathrm{V}$. Arnold. The answer for the case of $n=2$ can be found in the works of E. Korkina [3] and G. Lachaud [4]. In present work we study the case of $n=3$. The author is grateful to V. Arnold for constant attention to this work and useful remarks and Université Paris-Dauphine - CEREMADE for the hospitality and excellent working conditions.

Definitions. A point of $\mathbb{R}^{n+1}$ is called integer if all its coordinates are integers. A hyperplane is called integer if all its integer vectors generate $n$-dimensional sublattice of integer lattice. Consider some integer hyperplane and an integer point in the complement to this plane. Let the Euclidean distance from the given point to the given plane equals $l$. The minimal value of nonzero Euclidean distances from integer points of the space $\mathbb{R}^{n+1}$ to the plane is denoted by $l_{0}$. The ratio $l / l_{0}$ is said to be the integer distance from the given integer point to the given integer hyperplane.

Definition of multidimensional continued fraction in the sense of Klein. Consider arbitrary $n+1$ hyperplanes in $\mathbb{R}^{n+1}$ that intersect at the unique point: at the origin. Assume also that all the given planes do not contain any integer point different to the origin. The complement to these hyperplanes consists of $2^{n+1}$ open orthants. Consider one of the orthants. The boundary of the convex hull of all integer points except the origin in the closure of the orthant is called the sail of the orthant. The set of all $2^{n+1}$ sails is called the $n$-dimensional continued fraction constructed accordingly to the given $n+1$ hyperplanes. Two $n$-dimensional continued fractions are said to be equivalent if there exist a linear lattice preserving transformation of $\mathbb{R}^{n+1}$ taking all sails of one continued fraction to the sails of the other continued faction.

We associate to any hyperbolic irreducible operator $A$ of $S L(n+1, \mathbb{Z})$ an $n$-dimensional continued fraction constructed accordingly to the set of all $n+1$ eigen-hyperplanes for $A$. Any sail of such continued fraction is homeomorphic to $\mathbb{R}^{n}$. From Dirichlet unity theorem it follows that the group of all $S L(n+1, \mathbb{Z})$-operators commuting with $A$ and preserving the sails is homeomorphic to $\mathbb{Z}^{n}$ and its action is free (we denote this group by $\Xi(A)$ ). A fundamental domain of the sail with respect to the action of the group $\Xi(A)$ is a face union that contains exactly one face of the sail from each orbit. (For more information see [1], [2], [3] and [5].)

Date: 19 December 2005.

Key words and phrases. Integer lattice, continued fractions, convex hulls.

Partially supported by RFBR grant SS-1972.2003.1 and by RFBR grant 05-01-01012a. 
Denote by $A_{a, b, c, d}$ the following integer operator

$$
\left(\begin{array}{llll}
0 & 1 & 0 & 0 \\
0 & 0 & 1 & 0 \\
0 & 0 & 0 & 1 \\
a & b & c & d
\end{array}\right)
$$

Example 1. Consider the operator $A_{1}=A_{1,-3,0,4}$. The group $\Xi\left(A_{1}\right)$ is generated by the operators $B_{11}=A_{1}^{-2}, B_{12}=\left(A_{1}-E\right)^{2} A_{1}^{-2}$, and $B_{13}=\left(A_{1}-E\right)^{2}\left(A_{1}+E\right) A_{1}^{-2}$. Let us enumerate all three-dimensional faces for one of the fundamental domains of the sail containing the vertex $(0,0,0,1)$. Let $V_{10}=(-3,-2,-1,1), V_{1,4 i+2 j+k}=B_{11}^{i} B_{12}^{j} B_{13}^{k}\left(V_{10}\right)$ for $i, j, k \in\{0,1\}$. One of the fundamental domains of the sail contains the following three-dimensional faces: $T_{11}=V_{10} V_{12} V_{14} V_{15}, T_{12}=V_{12} V_{14} V_{15} V_{16}, T_{13}=V_{12} V_{15} V_{16} V_{17}$, $T_{14}=V_{12} V_{13} V_{15} V_{17}, T_{15}=V_{10} V_{12} V_{13} V_{15}, T_{16}=V_{10} V_{11} V_{13} V_{15}$, and $T_{17}=V_{10} V_{11} V_{12} V_{13}$ (see on the figure from the left). All listed tetrahedra are taken by some integer affine transformations to the unit basis tetrahedron. The integer distance from the origin to the planes containing the faces $T_{11}, \ldots, T_{17}$ equal $4,3,2,4,3,2$, and 1 , respectively.

Statement 1. The continued fraction constructed for any hyperbolic matrix of $S L(4, \mathbb{Z})$ with irreducible characteristic polynomial over rationals and with the sum of absolute values of the elements smaller than 8 is equivalent to the continued fraction of Example 1.

Statement 2. The symmetry (not commuting with $A_{1}$ ) defined by the matrix

$$
\left(\begin{array}{rrrr}
4 & -16 & 17 & -3 \\
3 & -11 & 11 & -2 \\
3 & -8 & 6 & -1 \\
6 & -8 & -2 & 1
\end{array}\right)
$$

acts on the sail of Example 1. This symmetry permutes the equivalence classes (with respect to the action of $\left.\Xi\left(A_{1}\right)\right)$ of tetrahedra $T_{11}$ and $T_{14}, T_{12}$ and $T_{15}, T_{13}$ and $T_{16}$, and takes the class of $T_{17}$ to itself.

Example 2. Let us consider the operator $A_{2}=A_{1,-4,1,4}$. The group $\Xi\left(A_{2}\right)$ is generated by the operators $B_{21}=A_{2}^{-2}, B_{22}=\left(A_{2}-E\right)^{2} A_{2}^{-2}$, and $B_{23}=\left(A_{2}+E\right) A_{2}^{-1}$. Let us enumerate all three-dimensional faces for one of the fundamental domains of the sail containing the vertex $(0,0,0,1)$. Let $V_{20}=(-4,-3,-2,0), V_{2,4 i+2 j+k}=B_{21}^{i} B_{22}^{j} B_{23}^{k}\left(V_{20}\right)$ for $i, j, k \in\{0,1\}$. One of the fundamental domains of the sail contains the following three-dimensional faces: $T_{21}=V_{20} V_{21} V_{23} V_{24}, T_{22}=V_{21} V_{23} V_{24} V_{25}, T_{23}=V_{20} V_{22} V_{23} V_{24}$, $T_{24}=V_{22} V_{23} V_{24} V_{26}, T_{25}=V_{23} V_{24} V_{25} V_{27}$, and $T_{26}=V_{23} V_{24} V_{26} V_{27}$ (see on the figure at the middle). All listed tetrahedra are taken by some integer affine transformations to the unit basis tetrahedron. The integer distance from the origin to the planes containing the faces $T_{21}, \ldots, T_{26}$ equal $1,2,2,4,8$, and 13 , respectively.

Example 3. Consider the operator $A_{3}=A_{-1,-3,1,3}$. The group $\Xi\left(A_{3}\right)$ is generated by the operators $B_{31}=A_{3}^{-2}, B_{32}=\left(A_{3}-E\right) A_{3}^{-1}$, and $B_{33}=A_{3}+E$. Any fundamental domain of the sail with $(0,0,0,1)$ as a vertex contains a unique three-dimensional face. The polyhedron $V_{30} V_{31} V_{32} V_{33} V_{34} V_{35} V_{36} V_{37}$ shown on the figure (the right one) is an example of such 
face, here $V_{30}=(-1,-1,-1,0), V_{31}=B_{33}\left(V_{30}\right), V_{32}=B_{32} B_{33}\left(V_{30}\right), V_{33}=B_{31} B_{32}^{-1}\left(V_{30}\right)$, $V_{34}=B_{32}^{-1}\left(V_{30}\right), V_{35}=B_{31} B_{33}^{2}\left(V_{30}\right), V_{36}=B_{31} B_{33}\left(V_{30}\right), V_{37}=B_{31} B_{32}^{-1} B_{33}\left(V_{30}\right)$. The described face is contained in the plane on the unit distance from the origin. The integer volume of the face equals 8 .

Example 3 provides the negative answer to the following question for the case of $n=$ 3: is it true, that any $n$-periodic $n$-dimensional sail contains an $n$-dimensional face in some hyperplane on integer distance to the origin greater than one? The answers for $n=2,4,5,6, \ldots$ are unknown. The answer to the following question is also unknown to the author: is it true, that any $n$-periodic $n$-dimensional sail contains an $n$-dimensional face in some hyperplane on unit integer distance to the origin?
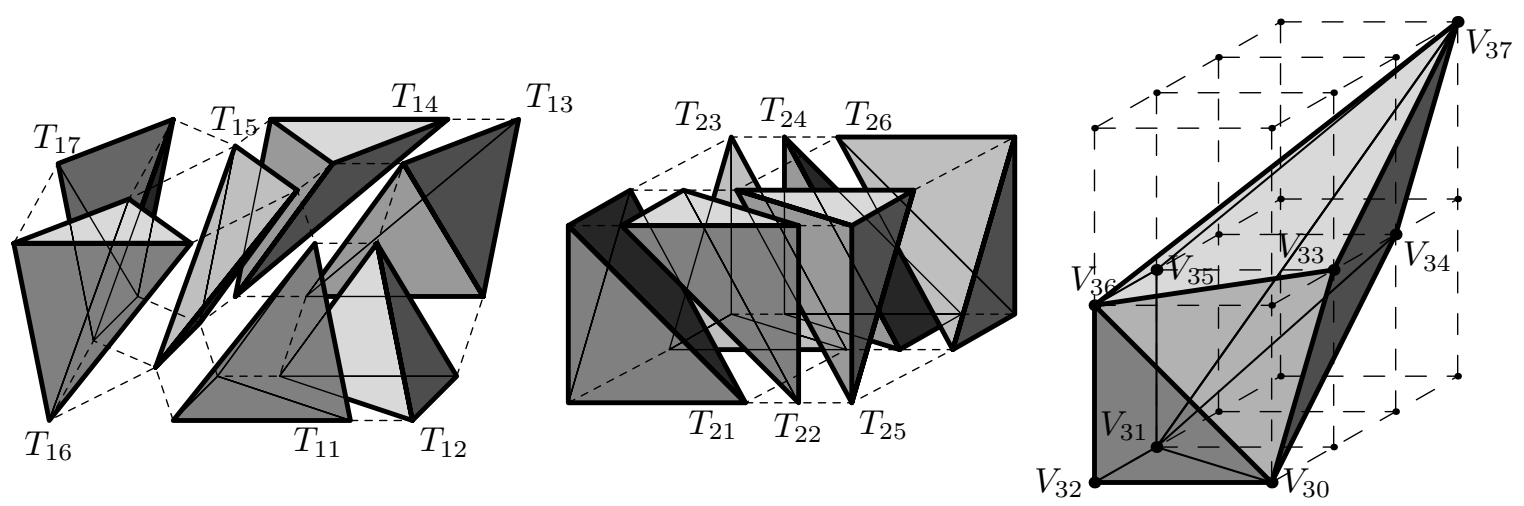

We show with dotted lines how to glue the faces to obtain the combinatorial scheme of the described fundamental domains.

\section{REFERENCES}

[1] V. I. Arnold, Continued fractions, M.: Moscow Center of Continuous Mathematical Education, 2002.

[2] O. N. Karpenkov, On tori decompositions associated with two-dimensional continued fractions of cubic irrationalities, Func. an. and appl., v. 38(2), pp. 28-37, (2004).

[3] E. I. Korkina, Two-dimensional continued fractions. The simplest examples, Proceedings of V. A. Steklov Math. Ins., v. 209(1995), pp. 143-166.

[4] G. Lachaud, Voiles et Polyèdres de Klein, preprint n 95-22, Laboratoire de Mathématiques Discrètes du C.N.R.S., Luminy (1995).

[5] J.-O. Moussafir, Voiles et Polyédres de Klein: Geometrie, Algorithmes et Statistiques, docteur en sciences thése, Université Paris IX - Dauphine, (2000).

E-mail address, Oleg Karpenkov: karpenk@ceremade.dauphine.fr

CEREMADE - UMR 7534 Université Paris-Dauphine France - 75775 Paris SEDEX 16 